Supplement of The Cryosphere, 14, 1747-1762, 2020

https://doi.org/10.5194/tc-14-1747-2020-supplement

(C) Author(s) 2020. This work is distributed under

the Creative Commons Attribution 4.0 License.

(c) (1)

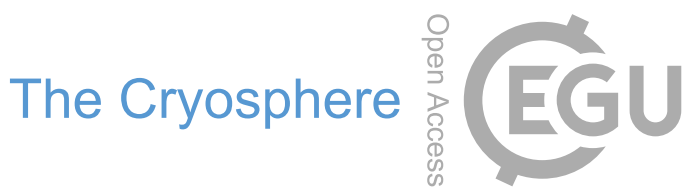

Supplement of

\title{
Remapping of Greenland ice sheet surface mass balance anomalies for large ensemble sea-level change projections
}

Heiko Goelzer et al.

Correspondence to: Heiko Goelzer (h.goelzer@uu.nl)

The copyright of individual parts of the supplement might differ from the CC BY 4.0 License. 


\section{Sensitivity to number of basins}

In order to test the sensitivity of the remapping to the number of basins, we have generated a schematic set of 20 basins that can be extended by subdivision to finer detail (Figure S3). The initial division (Fig. S3a) is created mostly by hand, first defining points on the main ice sheet divide (red) and their connectivity. From here the basins are delineated by connections

5 outwards along contour lines of equal surface elevation. Further sub-divisions (Fig. S3b,c) are added at equal distances along the surface contour arcs resulting in $20 \times(1+n)$ basins, where $n$ is an integer (here between 0 and 4). Compared to the basin set used throughout the rest of the manuscript, the delineations here do not follow established basin divides, which may be a limitation. We have therefore retained the physically-based basin delineation in the main text.
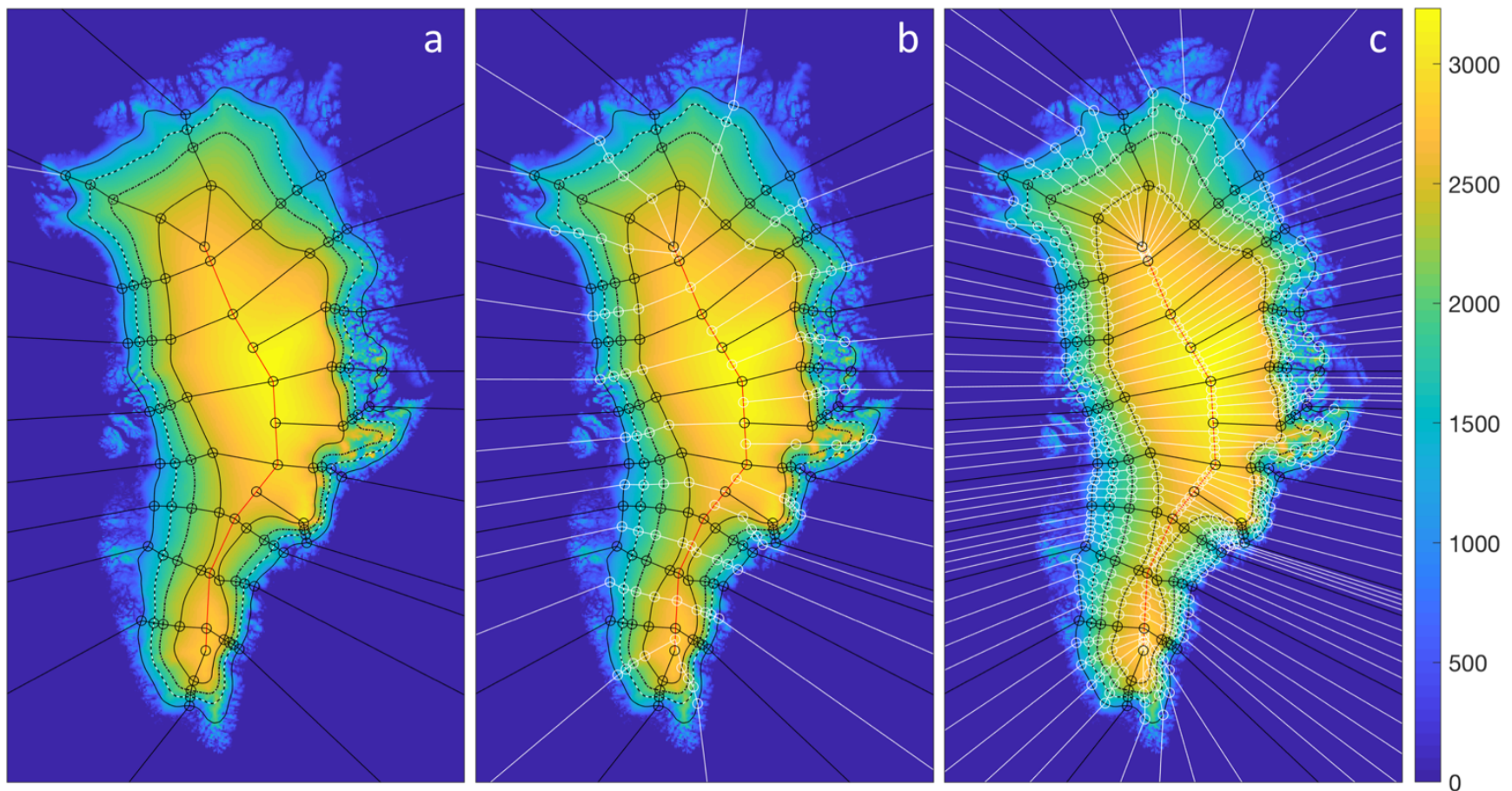

Figure S1 Semi-automatically generated division for testing sensitivity to number of basins.

[m]

We have calculated lookup tables and remapped SMB anomalies for 5 different basin numbers $(\mathrm{N}=20,40,60,80,100)$ and 6 different ISMIP6-Greenland GCMs (Goelzer et al., 2020). In some cases of very small regions (for large N) where no data

15 points fall into a specific elevation band, the lookup table information of the neighbouring basin is used. The resulting remapping error is given as root mean square error (RMSE) of the remapped compared to the original aSMB in Figure S4. In all cases, the lowest errors are attained with the highest number of basins $\mathrm{N}=100$, with steady decrease of the error towards larger $\mathrm{N}$ for most of the models. Differences in errors between lowest and highest $\mathrm{N}$ are on average 15 percent. 


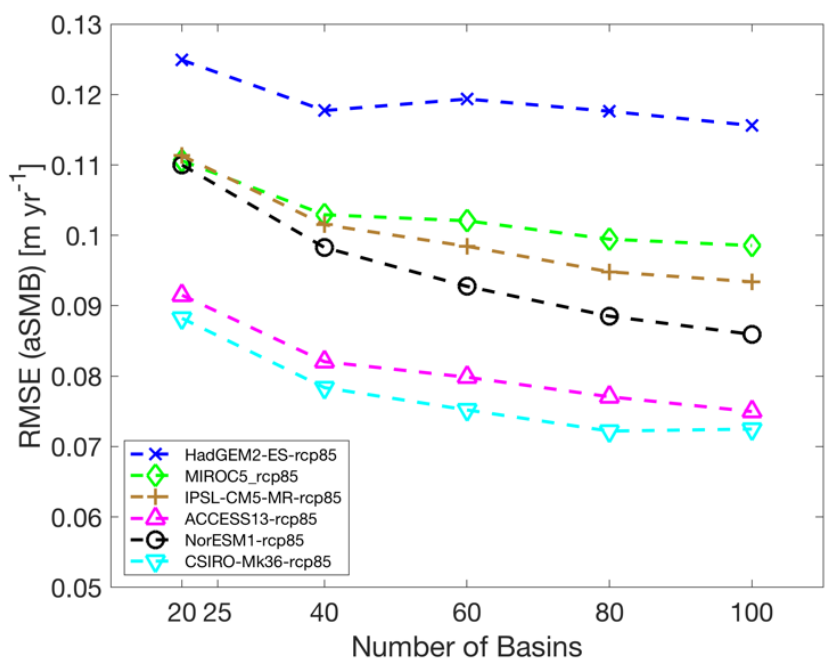

Figure S2 Root mean square error of remapped SMB anomaly as a function of the number of basins for MAR forced by 6 different CMIP5 GCMs.

\section{Results for additional model geometries}
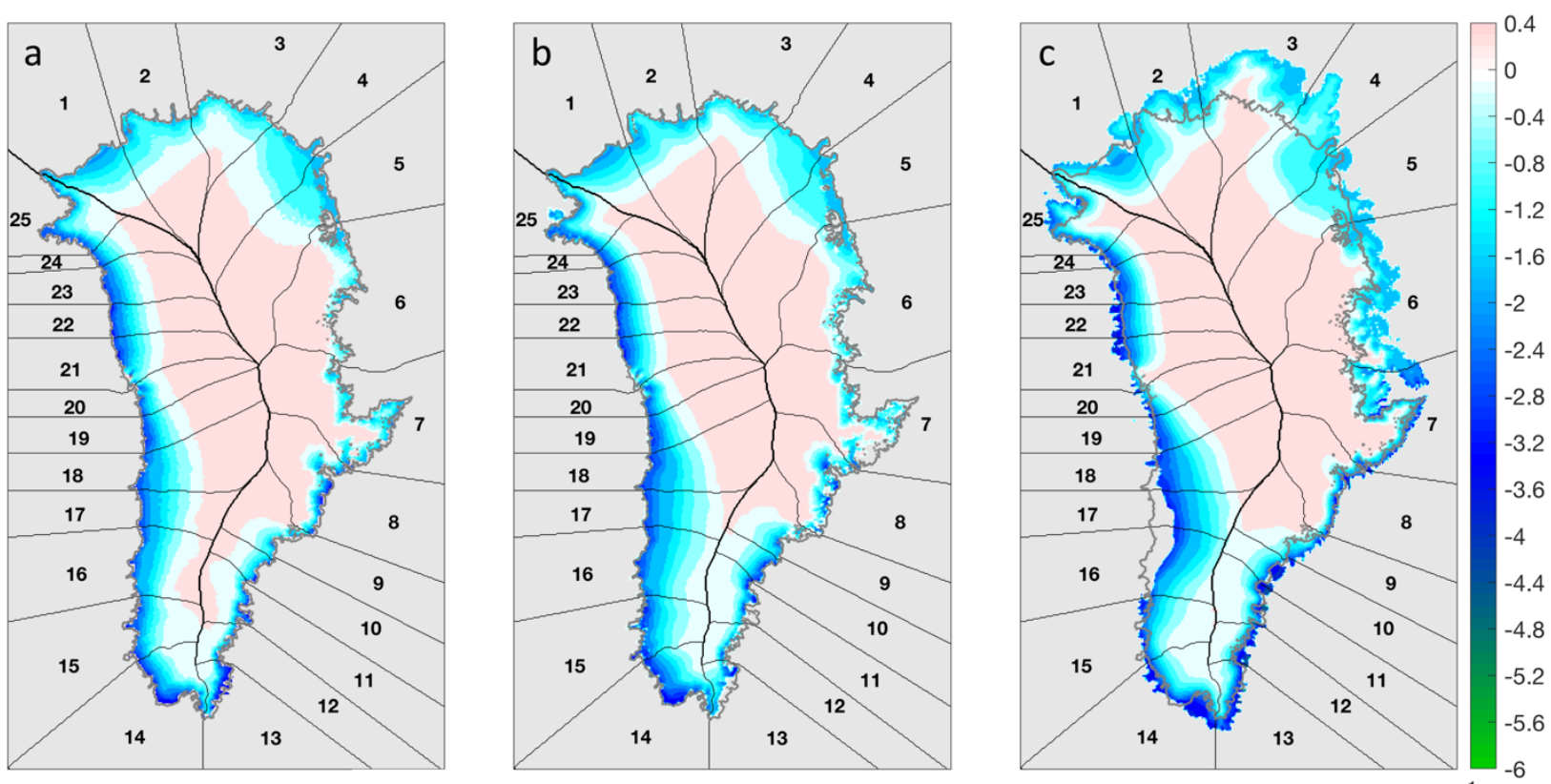

$\left[\mathrm{m} \mathrm{yr}^{-1}\right.$ ]

Figure S3 SMB anomaly from the RCM MAR remapped to the initial geometry of three different initMIP-Greenland models. Same as Figure 7c but for models JPL-ISSM (a), BGC-BISICLES (b) and MPIM-PISM (c). 

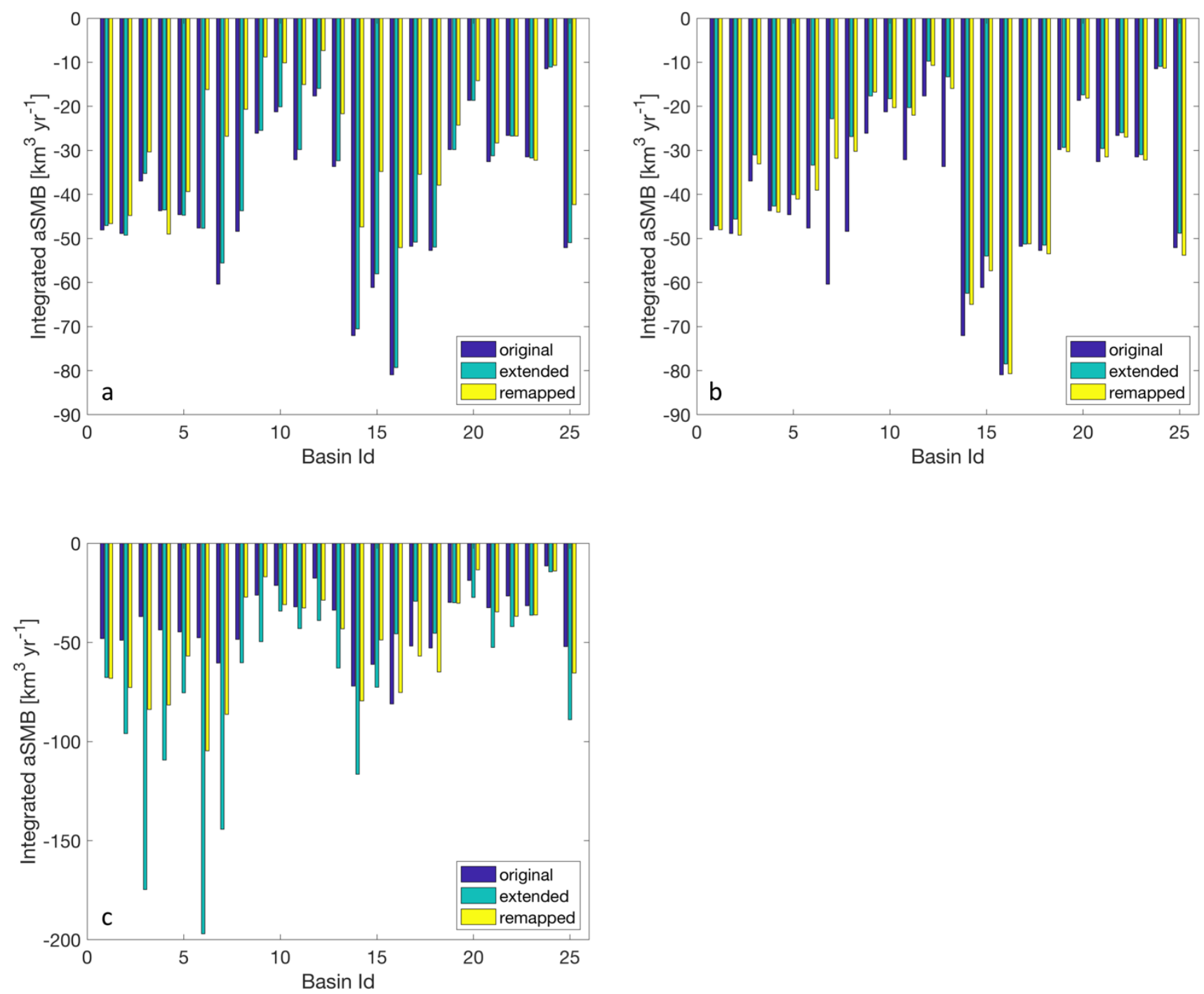

Figure S4 Integrated aSMB per basin from original MAR output (blue), when extended to the modelled ice mask (green) and for remapping to the modelled initial geometry from initMIP-Greenland (yellow): (a) JPL_ISSM, (b) BGC_BISICLES, (c) MPIM_PISM. Note differences in scale on the vertical axis between models. 